\title{
Measurement of high-mass dilepton production with the CMS-TOTEM Precision Proton Spectrometer
}

\author{
Ksenia Shchelina* ${ }^{\dagger}$ \\ Universita e INFN Torino (IT) \\ E-mail: k.shchelina@cern.ch
}

\begin{abstract}
The process $p p \rightarrow p \mu^{+} \mu^{-} p^{(*)}$ has been observed at the LHC for dimuon masses larger than $110 \mathrm{GeV}$ in $p p$ collisions at $\sqrt{s}=13 \mathrm{TeV}$. Here $p^{(*)}$ indicates the second proton is undetected, and either remains intact or dissociates into a low-mass state $p^{*}$. The scattered proton has been measured in the CMS-TOTEM Precision Proton Spectrometer (CT-PPS). The spectrometer operated for the first time in 2016, collecting $\sim 10 \mathrm{fb}^{-1}$ of data in regular, high-luminosity fills. The procedure for detector alignment, optics corrections, and data analysis including background estimate are described. The present data constitute the first evidence of this process at such masses. They also demonstrate that CT-PPS performs as expected.
\end{abstract}

The European Physical Society Conference on High Energy Physics

5-12 July

Venice, Italy

${ }^{*}$ Speaker.

${ }^{\dagger}$ On behalf of CMS and TOTEM Collaborations 


\section{Introduction}

The CMS+TOTEM Precision Proton Spectrometer (CT-PPS) [1] is a joint project of the CMS [2] and TOTEM [3] collaborations, aiming to operate near-beam forward proton detectors in high luminosity proton-proton running at the Large Hadron Collider (LHC). CT-PPS is designed for the study of the so-called "exclusive" reactions ( $p p \rightarrow p X p$ ), primarily via either $\gamma \gamma$ fusion, or gluongluon interactions, by detection of protons scattered at very small angles and carrying a substantial fraction of the incoming beam momentum. The detector consists of silicon tracking and fast timing detectors installed in Roman Pot stations $\sim 210-220 \mathrm{~m}$ from the CMS interaction point. The use of Roman Pot technology allows bringing the detectors very close (down to a few $\mathrm{mm}$ ) to the beam without affecting the accelerator vacuum.

CT-PPS operated for the first time in 2016 and collected a total of $\sim 15 \mathrm{fb}^{-1}$ in regular, highluminosity runs of LHC. Approximately $10 \mathrm{fb}^{-1}$ of these data, taken with Si-strips for tracking and before commissioning of the timing detectors, are studied for the analysis of central semiexclusive production of dimuon pairs, $p p \rightarrow p \mu^{+} \mu^{-} p^{(*)}$, with mass $M_{\mu \mu}>110 \mathrm{GeV}$. The two muons are measured in the central CMS apparatus, and the final state proton in the CT-PPS. Central semi-exclusive dimuon production has never been measured directly and is of interest because it constitutes a background to the exclusive reaction $p p \rightarrow p \mu^{+} \mu^{-} p$ when the final-state protons are not measured. In turn, the exclusive process is interesting because deviations from the expected cross section, which is well known, may be an indication of new physics. In central semi-exclusive events, the momentum of the two muons can be used to determine that of the proton, and hence the proton fractional momentum loss $\xi$. This measurement can be compared with the direct one obtained with CT-PPS. The comparison can be used to suppress background; it also provides proof of the correct functioning of CT-PPS and can be used to estimate its resolution.

\section{Alignment and optics corrections}

\subsection{Alignment}

The alignment is performed in two steps [10].

First, the data from a special, low-luminosity calibration fill are used to determine the absolute alignment. These data are collected with both horizontal and vertical RPs, inserted very close to the beam (about $5 \sigma$ ). The vertical pots are used to collect a sample of elastic scattering $p p \rightarrow p p$, allowing alignment with respect to the beam based on the azimuthal symmetry of this process. The horizontal RPs can then be aligned with respect to the vertical RPs, using a subsample of tracks passing through both.

The second step is the determination of the absolute alignment by transferring to the case of standard high-intensity fills. The procedure consists of matching the measured $\mathrm{x}$ distribution of track impact points, under the assumption that the same physics processes contribute to all fills. This results in a set of fill by fill alignment corrections, determined separately for each RP. The total uncertainty of the horizontal alignment procedure is on the order of $150 \mu \mathrm{m}$. The procedure is illustrated in Fig. 1. 


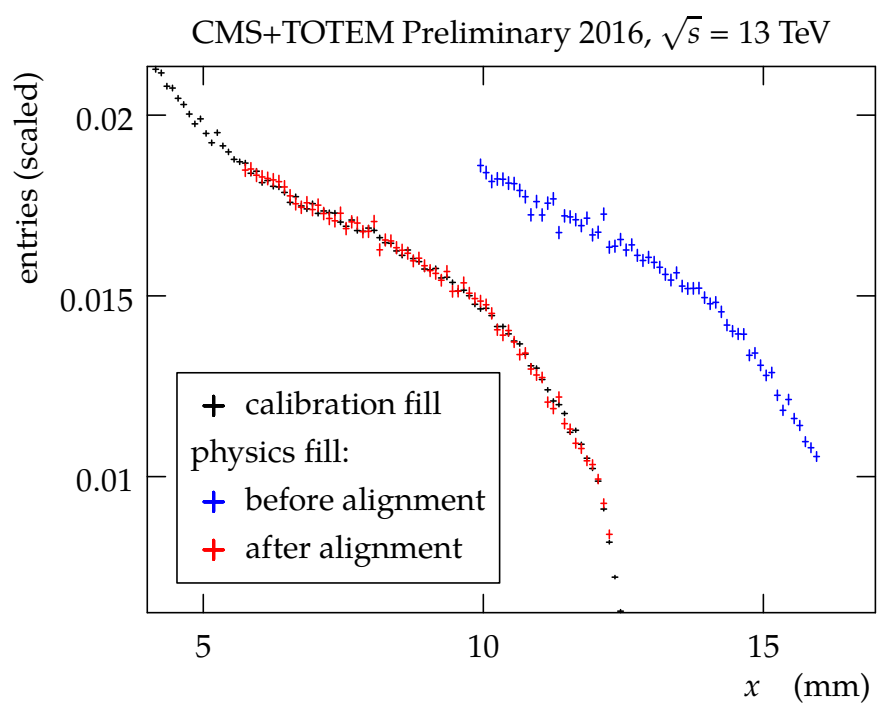

Figure 1: Distribution of the track impact points as a function of the horizontal coordinate for the alignment fill (black points), a physics fill before alignment (blue points), and after alignment (red points) [12].

\subsection{Optics}

A precise determination of the LHC beam optics is necessary to derive the proton's fractional energy loss $\xi$ from the measured x coordinates of the tracks after alignment. The procedure developed [11] allows to obtain data-driven constraints on the elements of the single pass transport matrix $T(s, \xi)$, whose elements are the optical functions of the beam line.

The leading horizontal term in the transport matrix is $\mathrm{x}=D_{x}(\xi) \xi$, where $D_{x}$ is the dispersion, which has a mild dependence on $\xi$. The leading term in the vertical plane is $y \approx L_{y}(\xi) \Theta_{y}^{*}$, where $L_{y}(\xi)$ is the vertical effective length and $\Theta_{y}^{*}$ is the vertical angle of the proton at the interaction point. The value of $L_{y}(\xi)$ will go to zero at a particular value of $\xi_{0}$, leading to a "pinch" in the vertical distribution of tracks reconstructed in the RP. By determining the horizontal position of this "pinch", the value of the dispersion can be obtained as $x_{0} \approx D_{x} \xi_{0}$ (Fig. 2), where higher-order terms are neglected and included in the systematic uncertainties.

A second independent method is also used to determine the difference in the dispersions in the two LHC beams, by comparing the measured physics proton distribution in the RPs.

\section{Analysis of $\gamma \gamma \rightarrow \mu^{+} \mu^{-}$production}

The alignment corrections and optics are applied for the analysis of $\gamma \gamma \rightarrow \mu^{+} \mu^{-}$production events [12], using $10 \mathrm{fb}^{-1}$ of data collected during 2016. Only one proton is required to be detected in CT-PPS in order to extend acceptance to the lower masses. This results in signal sample containing of a mix of both $p p \rightarrow p \mu^{+} \mu^{-} p$ and $p p \rightarrow p \mu^{+} \mu^{-} p^{*}$ events, in which one of the protons dissociates into an undetected system $p^{*}$. 


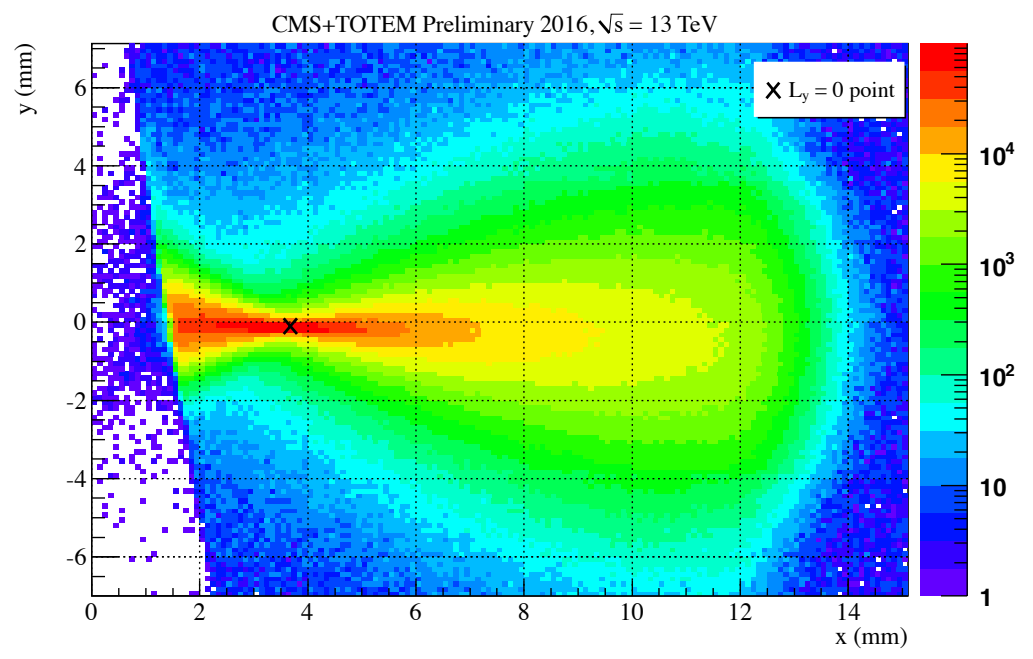

Figure 2: Distribution of the track impact points measured in RP 210F, in sector 45, in the alignment fill [12]. The point where $L_{y}=0$ and its effect in the impact point distribution are shown. The beam center is at $x=y=0$.

\subsection{Event selection and proton-dimuon matching}

In order to select a sample enriched in $\gamma \gamma \rightarrow \mu^{+} \mu^{-}$events, a similar procedure is used to that of the earlier Tevatron and LHC Run 1 LHC analyses where the proton tagging was not possible.

Events are required to have a dimuon vertex with no additional tracks within a veto region of $0.5 \mathrm{~mm}$. Both muons are required to have a transverse momentum $p_{T}>40 \mathrm{GeV}$, and invariant mass $m(\mu \mu)>110 \mathrm{GeV}$. In addition, the "acoplanarity" $(1-|\Delta \phi(\mu \mu)| / \pi)$ of the muons is required to be less than 0.009 . The selection criteria are chosen to obtain a signal to background ratio $>1$ after applying the central detector requirements. The selection is based on reconstructed track information and no calorimeter data because of the high rate of multiple collisions within the same bunch crossing ("pileup").

In the case of events in which both protons stay intact $\left(p p \rightarrow p \mu^{+} \mu^{-} p\right)$, the kinematics of the muons and the protons can be precisely related via the expression:

$$
\xi(\mu \mu)=\frac{1}{\sqrt{s}}\left(p_{T}\left(\mu_{1}\right) e^{ \pm \eta\left(\mu_{1}\right)}+p_{T}\left(\mu_{2}\right) e^{ \pm \eta\left(\mu_{2}\right)}\right) .
$$

If only one proton remains intact ( $p p \rightarrow p \mu^{+} \mu^{-} p^{*}$ ), the same expression approximately holds when the mass of the dissociating system $M_{X}$ is small; the deviation becomes comparable to the experimental dimuon resolution only for masses $M_{X} \geq 400 \mathrm{GeV}$. However, the expected fraction of such events is negligible because of the requirement of zero extra tracks.

The signal events are defined as those having $\xi(\mu \mu)$ and $\xi(\mathrm{RP})$ matching within $2 \sigma$ of their combined experimental resolution.

\subsection{Backgrounds and systematics}

After the central CMS detector selection, the backgrounds are expected to arise predominantly 
from Drell-Yan $\mu^{+} \mu^{-}$production and from $\gamma \gamma \rightarrow \mu^{+} \mu^{-}$production with both protons dissociating. These processes can mimic the signal when they overlap with the pileup proton or a Roman Pot track from beam-related backgrounds in the same bunch crossing. A control sample of $Z \rightarrow \mu^{+} \mu^{-}$events is used to estimate the probability of a background dimuon event overlapping with an uncorrelated RP track. The $\xi\left(\mu^{+} \mu^{-}\right)$distribution in the control region is reweighted to match the distribution predicted by the Drell-Yan simulation for events entering the signal region. For the double dissociation backgrounds, simulated events are normalized to the predicted number passing the central detector selection from simulation, and randomly mixed with protons from the $Z \rightarrow \mu^{+} \mu^{-}$data control sample.

Systematic uncertainties on the background yield include those arising from the statistical uncertainty in the $Z \rightarrow \mu^{+} \mu^{-}$sample used to estimate the backgrounds. For the Drell-Yan background, the systematic uncertainties due to the modeling of the track multiplicity in the simulation and the effect of reweighting the $\xi\left(\mu^{+} \mu^{-}\right)$distribution are also considered. For the double dissociation background, uncertainties in the integrated luminosity, and in the theoretical predictions of the survival probability [9] are included. The dominant uncertainties are due to the effect of reweighting the $\xi\left(\mu^{+} \mu^{-}\right)$distribution (25\%, taken as the full difference between the results with and without reweighting), and the modeling of the track multiplicity distribution (28\%, taken as the full difference between data and simulation in the region with 1-5 extra tracks at the dimuon vertex).

The event-by-event uncertainty on $\xi(\mu \mu)$ is estimated from simulation and is found to be $3.3 \%$. The number takes into account the data-simulation corrections derived from $Z \rightarrow \mu^{+} \mu^{-}$ events. The uncertainty on $\xi(\mathrm{RP})$ is taken to be $5.5 \%$, with the dominant source arising from the dispersion $D_{x}$ used for optics determination.

The total background estimate, including systematic uncertainties, is $1.47 \pm 0.06$ (stat.) \pm 0.52 (syst.) events, dominated by the Drell-Yan backgrounds.

\subsection{Results}

The correlation between the $\xi(\mu \mu)$ predicted from the dimuon system and the $\xi(\mathrm{RP})$ measured directly is shown in Fig. 3, for the two arms separately. The region of low $\xi(\mu \mu)$ is populated only by random background correlations, as any signal protons are expected to be outside of the RP acceptance. In the $\xi(\mu \mu)$ region compatible with the RP acceptance, 17 events are observed. Of these, 12 have $\xi(\mu \mu)$ and $\xi(\mathrm{RP})$ compatible within $2 \sigma$ of the resolution, compared to $1.47 \pm$ 0.06 (stat.) \pm 0.52 (syst.) such events expected from the backgrounds only. The significance for observing 12 matching events, including systematic uncertainties, is estimated to be $4.3 \sigma$.

Figure 4 shows the signal candidate events and the approximate CT-PPS acceptance in the rapidity-mass plane. The events are consistent with the acceptance for detecting one of the two protons in CT-PPS. No events with two detected protons are seen in the data. The highest mass candidate has $\mathrm{m}(\mu \mu)=341 \mathrm{GeV}$, below the region of acceptance for detecting both protons.

\section{Conclusions}

The $\gamma \gamma \rightarrow \mu^{+} \mu^{-}$process was studied with reconstructed forward protons in the CMS-TOTEM Precision Proton Spectrometer, using a sample of $\sim 10 \mathrm{fb}^{-1}$ collected in high luminosity $13 \mathrm{TeV}$ 

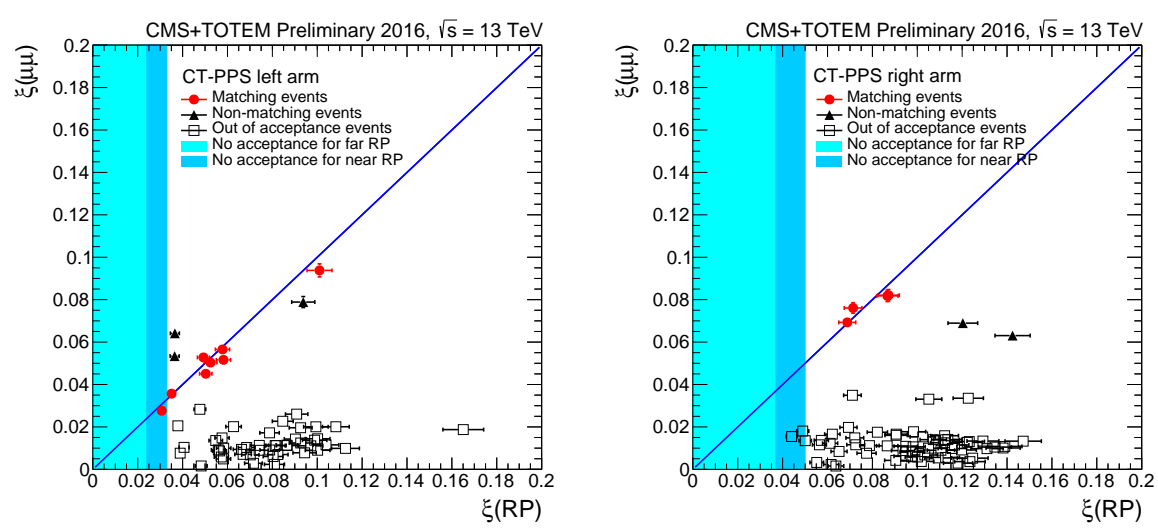

Figure 3: Correlation between $\xi(\mu \mu)$ and $\xi$ measured in the Roman Pots, for both Roman Pots in each arm combined [12]. The 45 (left) and 56 (right) arms are shown. The light shaded region corresponds to the kinematic region outside the acceptance of both the near and far RPs, while the darker shaded region corresponds to the region outside the acceptance of the near RP. For the events in which a track is detected in both, the $\xi$ value measured at the near RP is plotted.

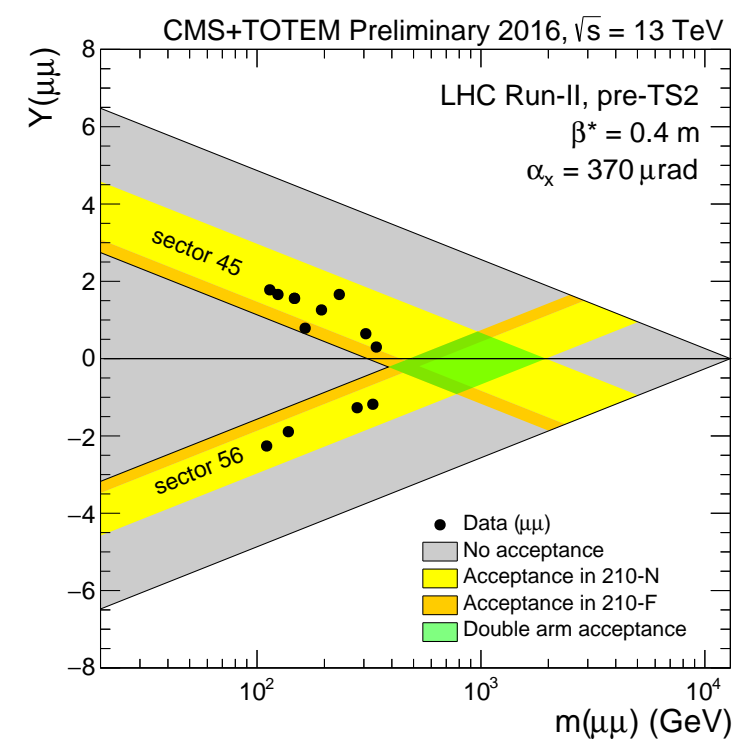

Figure 4: Expected approximate coverage in the rapidity vs invariant mass plane, overlaid with the observed dimuon signal candidate events [12].

LHC data taking. The Roman Pot alignment and LHC optics corrections have been derived independently and used for the physics analysis. A $>4 \sigma$ excess of $\gamma \gamma \rightarrow \mu^{+} \mu^{-}$candidates with $M_{\mu \mu}>110 \mathrm{GeV}$ and matching forward proton kinematics is observed, representing the first evidence for proton-tagged $\gamma \gamma$ collisions at the electroweak scale. With its 2016 operation, CT-PPS has proven for the first time the feasibility of operating a near-beam proton spectrometer at a high luminosity hadron collider on a regular basis. 


\section{References}

[1] M. Albrow et al., CERN-LHCC-2014-021, TOTEM-TDR-003, CMS-TDR-13 [http://cds.cern.ch/record/1973331].

[2] S. Chatrchyan et al. [CMS Collaboration], JINST 3, S08004 (2008). doi:10.1088/1748-0221/3/08/S08004

[3] G. Antchev et al. [TOTEM Collaboration], Int. J. Mod. Phys. A 28, 1330046 (2013) doi:10.1142/S0217751X13300469 [arXiv:1310.2908 [physics.ins-det]].

[4] J. de Favereau de Jeneret et al., arXiv:0908.2020 [hep-ph].

[5] K. Akiba et al. [LHC Forward Physics Working Group], J. Phys. G 43, 110201 (2016) doi:10.1088/0954-3899/43/11/110201 [arXiv:1611.05079 [hep-ph]].

[6] S. Fichet, G. von Gersdorff, B. Lenzi, C. Royon and M. Saimpert, JHEP 1502, 165 (2015) doi:10.1007/JHEP02(2015)165 [arXiv:1411.6629 [hep-ph]].

[7] S. Fichet, G. von Gersdorff, O. Kepka, B. Lenzi, C. Royon and M. Saimpert, Phys. Rev. D 89, 114004 (2014) doi:10.1103/PhysRevD.89.114004 [arXiv:1312.5153 [hep-ph]].

[8] S. Fichet, G. von Gersdorff and C. Royon, Phys. Rev. Lett. 116, no. 23, 231801 (2016) doi:10.1103/PhysRevLett.116.231801 [arXiv:1601.01712 [hep-ph]].

[9] L. A. Harland-Lang, V. A. Khoze and M. G. Ryskin, Eur. Phys. J. C 76, no. 5, 255 (2016) doi:10.1140/epjc/s10052-016-4100-2 [arXiv:1601.03772 [hep-ph]].

[10] J. Kaspar, CERN-TOTEM-NOTE-2017-001 (2017) [http://cds.cern.ch/record/2256296].

[11] F. Nemes, CERN-TOTEM-NOTE-2017-002 (2017) [http://cds.cern.ch/record/2256433].

[12] CMS Collaboration et al. [CMS and TOTEM Collaborations], CMS-PAS-PPS-17-001, TOTEM-NOTE-2017-003 [http://cds.cern.ch/record/2265781]. 